\title{
INCIDENCIA DE LA COMUNICACIÓN INTERNA CON LOS ESTUDIANTES DEL PROGRAMA SER PILO PAGA DE LA UNIVERSIDAD NACIONAL DE COLOMBIA, SEDE MEDELLÍN
}

\author{
Influence of internal communications with students in the program "Ser Pilo Paga" at \\ the National University of Colombia in Medellin
}

Luis Eduardo Cárdenas-Valencia

Magíster en Comunicaciones, docente Universidad de Antioquia, Medellín-Colombia, ps.eduardo.cardenas@gmail.com

\section{Blanca Deyanira Aguilar-Vélez}

Magíster en Psicología, docente Universidad de Antioquia y Universidad Pontificia Bolivariana, MedellínColombia, deyaniraclases@gmail.com

\begin{abstract}
Cómo referenciar / How to cite
Cárdenas-Valencia, L., \& Aguilar-Vélez, B. (2018). Incidencia de la comunicación interna con los estudiantes del programa Ser Pilo Paga de la Universidad Nacional de Colombia, sede Medellín. Revista CEA, 4(8), 35-49. https://doi.org/10.22430/24223182.1046
\end{abstract}

Recibido: 5 de febrero de 2018

Aceptado: 7 de mayo de 2018

\section{Resumen}

La comunicación interna en las universidades suele estudiarse a partir de las relaciones laborales establecidas con su personal administrativo y docente; sin embargo, las interacciones que tienen lugar con y entre los estudiantes como su público más representativo evidencian la complejidad de este tipo de organizaciones para el logro de sus objetivos.

Este artículo ofrece una reflexión en torno a la investigación mixta de dos fases con preponderancia cualitativa, que tiene como objetivo analizar la incidencia de la comunicación interna con los estudiantes del programa Ser Pilo Paga en la formación de su cultura organizacional en la Universidad Nacional de Colombia, sede Medellín; programa que surge como iniciativa del Gobierno Nacional y que opera a partir de las dinámicas propias de cada institución de Educación Superior, por lo que es de vital importancia conocer acerca de las interacciones que surgen con sus miembros, así como las representaciones y significados que se construyen a partir de su pertenencia al programa y a la Universidad para el logro de los objetivos e intenciones que los atraviesan.

Palabras clave: comunicación interna en universidades, cultura organizacional, investigación de comunicación en universidades, programa Ser Pilo Paga.
Abstract
Internal communications at universities are usually studied from the standpoint of the work relationships with its administrative staff and faculty. However, the interactions with and among students, their most representative audience, reveal the complexity of this type of organizations.
This study adopted a two-phase mixed methods approach with qualitative preponderance to analyze the influence of internal communications with students in the program "Ser Pilo Paga" on the development of their organizational culture at the National University of Colombia in Medellín. Said program is as an initiative of the National Government and it works based on the 
dynamics of each higher education institution. As a result, to achieve their common goals, the interactions between its members, as well as the representations and meanings that they build on their sense of belonging to the program and the University, must be examined.

Keywords: Internal communications, universities, organizational culture, communication research, Ser Pilo Paga.

\section{INTRODUCCIÓN}

Ser Pilo Paga surge como iniciativa entre el Ministerio de Educación Nacional de Colombia y el ICETEX ${ }^{1}$, un programa cuya finalidad es la de promover el acceso a la Educación Superior a los estudiantes con bajos recursos económicos que contaron con altos puntajes en las pruebas Saber $11^{2}$, a partir de créditos condonables que se han otorgado desde el año 2015³. El Gobierno Nacional cubre la totalidad de la matrícula de los estudiantes adscritos al programa y ofrece recursos que cubren una parte de su sostenimiento; además, asigna presupuesto a las universidades por cada estudiante del programa que se encuentra matriculado en ellas.

Desde el año 2015 hasta la mitad del segundo semestre de 2016, los estudiantes Ilamados Pilos de la Universidad Nacional de Colombia, sede Medellín, fueron parte de la comunidad estudiantil como cualquier miembro regular, sin intervenciones desde Ser Pilo Paga por medio de actividades especiales en la universidad, pero sí recibiendo los beneficios económicos asociados con su pertenencia al programa. De este modo, los imaginarios,

\footnotetext{
${ }^{1}$ El ICETEX es una entidad estatal de Colombia encargada de otorgar créditos educativos para promover la Educación Superior de las personas con necesidades económicas y buen desempeño académico.

2 Estas pruebas se han realizado en las Instituciones de Educación Media de Colombia a los estudiantes de Undécimo grado, encargándose de monitorear la calidad de la educación de los colegios del país y comprobar cómo se encuentran las
}

significados, ideales y temores que se construyeron en torno a Ser Pilos devenían principalmente de las relaciones familiares y personales, mas no de las interacciones generadas con otros miembros del programa ni con profesionales que tuvieran el rol de representarlo en la universidad.

Las actividades dirigidas a esta población, desde el sistema de Bienestar Universitario, se iniciaron a mediados del segundo semestre académico del año 2016, atendiéndose necesidades psicosociales y académicas de esta población, que de igual forma benefició a los estudiantes regulares que desearon participar. Antes del establecimiento de la responsabilidad sobre Ser Pilo Paga al área de Acompañamiento Integral de la universidad, la comunicación del programa se enfocaba en transmitir información asociada con los requisitos de la convocatoria, recordatorios de las fechas de entrega de documentación importante e invitaciones a participar de actividades eventuales de orden nacional.

Para el planteamiento investigativo se consideró este contexto con la particularidad de los ejes con que se estableció el programa en la sección de Acompañamiento Integral del sistema de Bienestar Universitario de la Universidad Nacional de Colombia, Sede Medellín; en donde uno de sus enfoques de trabajo se ha declarado hacia la articulación de líderes de la Sociedad Pilo, la cual no contaba con acciones para su conformación o mantenimiento cuando el programa inició su ejecución en el país en el año 2015.

Al relacionarse este asunto con la ausencia de un plan operativo enfocado en el programa, sino hasta finales del 2016, surgió la pregunta

competencias de los estudiantes que finalizan la educación media.

${ }^{3}$ Estos créditos cubren el $100 \%$ de los gastos de la matrícula siempre y cuando el estudiante culmine con sus estudios en los tiempos requeridos por cada versión de Ser Pilo Paga, de lo contrario, el valor debe ser cancelado por el estudiante y su familia. 
de investigación respecto a cómo ha incidido la comunicación interna con los estudiantes del programa Ser Pilo Paga en la formación de su cultura organizacional en la Universidad Nacional de Colombia, sede Medellín; interrogante que se abordó por medio de una investigación mixta, de preponderancia cualitativa, de dos fases secuenciales de recolección de información. En la primera fase, de carácter cuantitativo, se realizó un estudio de método no experimental de manera transversal a finales del año 2016, información que requirió ser profundizada por medio de la segunda fase, de orden cualitativo, que tuvo lugar en el primer semestre del año 2017 a partir del método hermenéutico.

El proceso investigativo que tuvo como objetivo general analizar la influencia de la comunicación interna con los estudiantes del programa Ser Pilo Paga en la formación de su cultura organizacional, se llevó a cabo a partir de sus tres objetivos delimitados en: 1) estimar elementos de la comunicación interna y la cultura organizacional a partir de los usuarios pertenecientes al programa; 2) identificar las características de la comunicación interna y la cultura organizacional en torno a los significados, creencias, valores y comportamientos de los estudiantes Pilos de la sede; y 3) establecer los efectos de la comunicación interna orientada al programa sobre su cultura organizacional.

Esta investigación se realizó en la Sede Medellín de la Universidad Nacional de Colombia desde finales del año 2016 y finales del primer semestre del 2017, a partir de una encuesta estructurada de escala Likert dirigida a los estudiantes adscritos a Ser Pilo Paga de esta sede universitaria y entrevistas semiestructuradas dirigidas a estudiantes Pilos pertenecientes a las cinco facultades de la sede y a las dos primeras cohortes del programa, así como a profesionales en comunicación y a personal administrativo relacionados con los estudiantes o la ejecución del programa.

Considerando las dos unidades de análisis correspondientes a la comunicación interna y la cultura organizacional, se establecieron las variables y categorías con base en la revisión bibliográfica y la información obtenida con los instrumentos aplicados, por medio del software Atlas.ti, las cuales abarcaron las temáticas de flujos, contenidos e interpretaciones en torno a la comunicación interna, y de relaciones, significados y asociadas al componente simbólico de la cultura organizacional sobre los valores y creencias.

Las reflexiones de este ejercicio investigativo se presentarán luego de una exposición del desarrollo metodológico, el cual se enfocó en comprender la incidencia de la comunicación interna en la cultura organizacional y permitió cuestionar el lugar que tiene la comunicación en las universidades, así como su aporte en la ejecución de programas de intervención enfocados en la comunidad estudiantil. Es así como el aporte investigativo recae sobre la reflexión sobre la teoría y la metodología del estudio de la comunicación en las organizaciones y en la comprensión del papel de la comunicación en la ejecución de programas de orden estatal en las universidades.

El análisis que deviene con esta investigación da lugar a pensar en el rol de la comunicación a la hora de establecer programas dentro de organizaciones complejas, con públicos y objetivos heterogéneos, como lo son las universidades. Asimismo, la reciente implementación de Ser Pilo Paga como propuesta del Gobierno Nacional de Colombia hacia las instituciones de educación superior, es un escenario propicio para estudiar la importancia de la comunicación a la hora de efectuar programas que articulen contextos a nivel nacional. 


\section{METOdOLOGÍA}

Dado que Ser Pilo Paga hace parte de una iniciativa de carácter nacional, la investigación se enfocó en la particularidad del caso de la sede Medellín de la Universidad Nacional de Colombia, con el fin de comprender las características comunicacionales y sus repercusiones sobre la cultura organizacional del programa, más allá de intentar generalizar los resultados hacia las instituciones de Educación Superior en que también se ha ejecutado.

Para llevar a cabo este ejercicio investigativo se implementó una metodología mixta que, dentro de la investigación social, propende por la complementariedad al apoyarse de la diversidad de técnicas enfocadas en un mismo objeto de estudio, con el fin de aprehender la complejidad de la realidad (Blanco \& Pirela, 2016). Los recursos a partir de los cuales se apoya la investigación multimétodo, también conocida como «enfoque múltiple, diseño mixto, integrado, multimodal o multi estrategia» (p. 102), se centran en la determinación de los métodos más convenientes a partir de un carácter sistemático, lógico y justificado en función de las características de la investigación y el problema a estudiar (Senior, Colina, Marín, \& Perozo, 2012).

De este modo, se integraron elementos cuantitativos y cualitativos en la investigación, con el fin de complementar los resultados de la encuesta, propia de la fase cuantitativa, a partir de la profundización cualitativa en la realización de entrevistas semiestructuradas y llegar así a comprender en mayor medida la realidad social que motiva el estudio, por medio de la triangulación de métodos y de los resultados de las unidades de análisis (Blanco \& Pirela, 2016).

En esta metodología, para el proceso de recolección de información, se inició con la estimación de variables concernientes a las unidades de análisis principales: comunicación interna y cultura organizacional, por medio de una encuesta estructurada de escala Likert dirigida a una muestra representativa de estudiantes Pilos. Posteriormente, estos resultados cuantitativos se profundizaron con la obtención de información cualitativa por entrevistas semiestructuradas aplicadas a algunos estudiantes Pilos seleccionados, a profesionales asociados con el programa o con la sede universitaria relacionados con las unidades de análisis.

\subsection{Fase cuantitativa}

La totalidad de estudiantes adscritos a Ser Pilo Paga para finales del año 2016, en la sede Medellín de la Universidad Nacional de Colombia, era de 264 Pilos matriculados; con esto, se realizó el cálculo para determinar la muestra probabilística representativa de ellos a partir de la Ecuación 1 para población finita:

Ecuación 1:

$n=\frac{N * \mathrm{Z} \alpha^{2} * \mathrm{p} * \mathrm{q}}{e^{2}(N-1)+\mathrm{Z} \alpha^{2} * p * q}$

Contando con una población total de 264 Pilos de las dos primeras cohortes del programa en la Universidad Nacional, sede Medellín $(N)$, se debió seleccionar al menos una muestra de 133 integrantes del programa (n) para calcular una proporción esperada del $5 \%$ ( $p$, para lo cual $q=0,95)$ con un error aceptado (o precisión) del $6 \%$ (e) y un nivel de confianza del $95 \%(Z$, donde $Z \alpha$ equivale a 1,96).

De este modo, contando con estas características, las encuestas se aplicaron a 134 estudiantes pertenecientes al programa Ser Pilo Paga en la sede, indagándose acerca de la valoración considerada para el relacionamiento horizontal y vertical en cuanto a la comunicación, las características de la comunicación establecida, interacciones, participación, sentido de 
pertenencia e interés en ser parte activa del programa en actividades extracurriculares.

\subsection{Fase Cualitativa}

Posterior al componente cuantitativo, para la selección de participantes en la segunda fase se consideraron a quienes pudieran aportar información desde sus experiencias y representaciones, no solo con su pertenencia o relación directa con el programa Ser Pilo Paga, sino por el hecho de conocer respecto a las dinámicas de carácter comunicacional y administrativo en la sede, que pudieran ofrecer mayor profundidad en la información recolectada.

Con el fin de obtener nociones, tanto de las características de la comunicación interna como de los significados construidos alrededor de la cultura organizacional por parte de los estudiantes pertenecientes al programa Ser Pilo Paga y sus interacciones con la comunidad universitaria, la selección se realizó de manera dirigida (no probabilística) y variada para abarcar diferentes características de la población, buscando dar cuenta de la complejidad del fenómeno y enfocarse en los casos reconocidos como usuales en el contexto a investigar (Quintana, 2006), así como «mostrar distintas perspectivas y representar la complejidad del fenómeno estudiado, o bien documentar la diversidad para localizar diferencias y coincidencias, patrones $y$ particularidades» (Hernández-Sampieri, Fernández, \& Baptista, 2014, p. 387). De este modo, los participantes fueron agrupados en: estudiantes adscritos al programa Ser Pilo Paga, comunicadores y personal administrativo relacionado con el programa.

Se obtuvo, de este modo, la participación de seis estudiantes pertenecientes a Ser Pilo Paga que contaron con variedad en el sexo, en sus características académicas al pertenecer a las diferentes facultades de la sede, en contar con un promedio diverso, así como provenir de diferentes partes del país y del departamento de Antioquia.
Para la selección de los comunicadores como informantes clave, se tomó como indicio la entrevista realizada en la prueba piloto al jefe de la Unidad de Medios de Comunicación (Unimedios), con el cual se identificaron las características administrativas sobre cómo opera la comunicación en la sede y se corroboró la información declarada en la política de comunicación por parte de la Rectoría con la resolución 101 del 10 de febrero de 2016, donde se define la comunicación como un mecanismo de regulación social que trasciende la idea de medios y la reconoce como elemento fundamental para lograr sus fines.

Asimismo, con el fin de comprender con mayor detalle las características organizacionales y la representación frente a la comunicación en la sede y el programa, más allá de las concepciones y el quehacer de los comunicadores, se seleccionaron cuatro individuos que han aportado desde su ejercicio profesional a la comprensión de las dinámicas organizacionales y la comunicación directa con estudiantes adscritos a Ser Pilo Paga como personal administrativo de la Sede.

Es así como la investigación contó con la participación de 12 sujetos, entre los estudiantes Pilos, comunicadores y personal administrativo, información visualizada en la Tabla 1.

\subsection{Instrumentos de recolección de información cualitativa}

Considerando a la comunicación interna y la cultura organizacional como las unidades de análisis que orientaron la investigación, se diseñaron entrevistas semiestructuradas para los tres grupos de individuos que participaron en esta fase del proceso de recolección de información (estudiantes, comunicadores y personal administrativo). La comunicación interna se analizó en los tres grupos de interés para la investigación debido a las opiniones, percepciones, experiencias y conocimientos de las 
interacciones y los procesos comunicativos vivenciados; en cambio, la cultura organizacional fue estudiada únicamente con los estudiantes pertenecientes a Ser Pilo Paga, enfocándose en los significados, creencias, valores y comportamientos establecidos y construidos como integrantes del programa. Debido a que no ha sido explícita una cultura organizacional declarada desde el carácter institucional, el interés investigativo se enfocó en las representaciones y significados construidos a partir de las interacciones suscitadas en torno a la vivencia de Ser Pilo Paga en la Sede Medellín de la Universidad Nacional de Colombia.

Tabla 1. Sujetos de investigación seleccionados

Table 1. Selected research subjects

\begin{tabular}{ll}
\hline Categorías & Sujetos de investigación \\
\hline Comunicadores & 1 comunicadora de la Unidad de Medios de Comunicación \\
& 1 comunicadora Bienestar Universitario \\
\hline & 1 psicólogo sección de Acompañamiento Integral \\
& 1 profesional de trámites ICETEX sección de Gestión y Fomento \\
& Socioeconómico \\
& 1 jefe de la sección de Acompañamiento Integral \\
& 1 profesor jubilado - asesor Bienestar Universitario \\
\hline & 1 estudiante de la Facultad de Arquitectura \\
& 2 estudiantes de la Facultad de Ciencias ${ }^{4}$ \\
& 1 estudiante de la Facultad de Ciencias Agrarias \\
Estudiantes adscritos al & 1 estudiante de la Facultad de Ciencias Humanas y Económicas \\
programa Ser Pilo Paga & 1 estudiante de la Facultad de Minas \\
\hline
\end{tabular}

Fuente: Elaboración propia.

\section{RESULTADOS}

La comunicación interna, componente organizacional que se ha posicionado como elemento estratégico en los últimos años, al reflejar la dependencia existente entre los empleados y la organización para la materialización de sus estrategias (García, Ruiz, \& Ventura, 1999; Constantin \& Constantin, 2015), además de alinear las metas entre los agentes de la organización, promover el entendimiento misional y la configuración de un clima adecuado entre los colaboradores (Ruck \& Welch, 2012; Bartels, Peters, Jong, Pruyn \& van der Molen, 2010), permite la interacción entre los miembros, promueve las identificaciones y establece un campo a partir del cual se construyen, representan y transforman las realidades que comparten quienes hacen parte de la cotidianidad organizacional (Fajardo, 2009; Saladrigas, 2005; Tapia \& Matellanes, 2009). Así, desde las formas en que la comunicación se instaura en las organizaciones, se propende por la construcción del sentido y los significados por medio de las relaciones y el valor que los miembros les atribuyen en el devenir de la cotidianidad organizacional (Karanges, Johnston, Beatson, \& Lings, 2015).

De este modo, dentro de las características de la comunicación interna en el programa

\footnotetext{
${ }^{4}$ Uno de estos estudiantes manifestó abiertamente no encontrarse a gusto en su programa académico, por lo que para el 2017 realizó cancelación de su pertenencia al programa Ser Pilo Paga.
} 
Ser Pilo Paga de la Universidad Nacional de Colombia, Sede Medellín, se presenta la prevalencia del flujo descendente de la comunicación, con el uso de los medios institucionales de la sede y las interacciones que tienen lugar en su interior. Esto dio lugar a fomentar una función informativa unidireccional de la comunicación y posibilitar pocos mecanismos para la presencia de flujos ascendentes, desde los estudiantes hacia estancias superiores, y horizontales, entre ellos, por lo que sus iniciativas fueron escasas a la hora de buscar comunicarse con sus superiores o de generar interacciones entre sí. De este modo, la comunicación se centra en entregar información desde las estancias encargadas de gestionar y dirigir, limitando la participación en la toma de decisiones, así como la mediación entre las estancias superiores e inferiores más allá de la emisión de contenidos institucionales y operativos (Bartels, Peters, Jong, Pruyn, \& van der Molen, 2010; Preciado-Hoyos \& Etayo-Pérez, 2014).

Los contenidos de carácter descendente recibidos por los estudiantes, a la hora de hacer explícita la procedencia de Ser Pilo Paga hizo que fueran atendidos más fácilmente por sus integrantes, al contrario de lo ocurrido con otros contenidos de la universidad que no han sido de su interés, como los mensajes enviados por otras facultades, temáticas de condolencias, novedades de otras sedes o de otras universidades, entre otros contenidos. Es así como los mensajes rotulados con el nombre del programa dan pie a ser considerados de mayor importancia, excepto cuando las convocatorias hacia los Pilos no explicitan obligatoriedad o algún trámite que ponga en riesgo su permanencia en el programa; de este modo, la comunicación predominantemente es formal y de carácter institucional, y no se presentan contenidos emitidos desde los estudiantes para promover las interacciones, el apoyo y el soporte entre sí, propio de los flujos horizontales (Bartels, Peters, Jong, Pruyn \& van der Molen, 2010).

Esto se refuerza con la intención comunicacional de la universidad en torno a unificar, preservar y custodiar la imagen institucional en los contenidos que circulan por los canales formales, orientándose principalmente a las dependencias académicas y administrativas que requieran comunicarse con la comunidad universitaria. Es así como el rol de los estudiantes se posiciona bajo la pasividad, al ser miembros que mencionan no disponer del conocimiento acerca de las formas para lograr ascender la información o el interés en hacerlo, así como la falta de canales formales para interactuar entre sí.

Los medios digitales tienen mayor predominancia a la hora de establecer relación con la población estudiantil de orden descendente, siendo el correo electrónico aquel que presenta mayor actividad a la hora de emitir información; no obstante, su saturación por el exceso de mensajes da lugar a un bajo interés por consumir todos sus contenidos. Además, si bien se conoce la existencia de otros medios virtuales propios de la universidad y sus dependencias, los estudiantes prefieren recurrir a medios informales, como lo son perfiles no oficiales en redes sociales, para establecer contacto, resolver inquietudes y obtener mayor detalle sobre aquello que informa la universidad, puesto que se reconoce que la comunicación descendente no suele habilitar mecanismos de retroalimentación por parte de los estudiantes receptores $y$, por lo tanto, se presenta una baja involucración y compromiso, lo cual es un asunto vital para la vida organizacional y la calidad de su relacionamiento (Moreno, Molleda, Athaydes, Suárez, Herrera, \& Álvarez, 2017).

La escasez de bidireccionalidad y de retroalimentación en la comunicación descendente, propias de la comunicación institucional, se transforma conforme se genera una mayor aproximación a los 
públicos de interés por medio de actividades que facilitan los mecanismos para ser escuchados. De esta manera, las actividades del Sistema de Bienestar Universitario, principalmente desde sus áreas y programas, así como la operación de Ser Pilo Paga por labor de la sección de Acompañamiento Integral, establecen mecanismos donde la comunicación descendente promueve las actividades en que los asistentes pueden participar, privilegiándose posteriormente la comunicación cara a cara $y$, con ello, la posibilidad de ser escuchados y relacionarse con sus pares. No obstante, debido a que la presencialidad es un componente esencial a la hora de lograrse la interacción y la retroalimentación de esta forma de comunicación, la falta de interés y la inasistencia afectan la eficiencia de estos medios de relacionamiento. Parte de los causantes que dificultaron el establecimiento de la comunicación ascendente, de los estudiantes Pilos con estancias superiores, fue el hecho de requerir de su iniciativa a la hora de buscar los mecanismos para dar a conocer sus ideas, el desconocimiento de los medios para hacerlo, la desesperanza frente a ser realmente escuchados, así como la incomodidad que ha generado para ellos sentirse involucrados o catalogados como quienes expresan la inconformidad o las quejas, como «revolucionarios».

La predominancia del flujo descendente unidireccional de la comunicación, así como la poca interacción ascendente y horizontal por el bajo interés de los estudiantes en promover este tipo de relacionamiento (Figura 1), dan lugar al desconocimiento por parte de las entidades superiores acerca de las experiencias y representaciones construidas por los pilos de la sede, construyéndose en ellas una imagen distorsionada y poco acorde con la realidad organizacional (Preciado-Hoyos \& EtayoPérez, 2014).

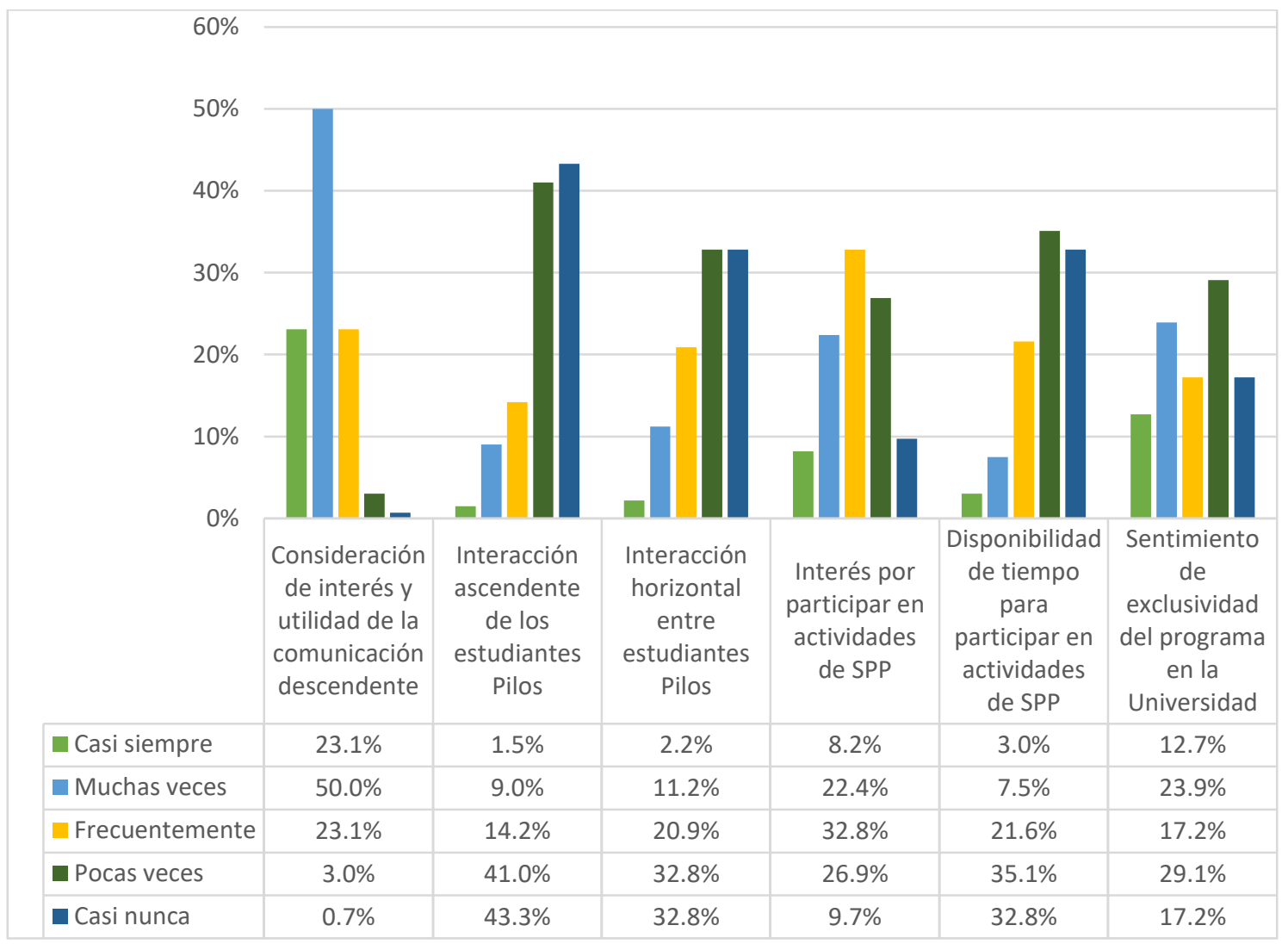

Figura 1. Estimación de la comunicación y participación de los estudiantes Pilos

Figure 1. Evaluation of communications and participation by students in "Ser Pilo Paga" Fuente: Elaboración propia. 
Aunque la comunicación establecida desde la universidad con los estudiantes Pilos es considerada igual a aquella que se presenta con el resto de la comunidad estudiantil, donde predomina la función informativa y descendente unidireccional, los Pilos referencian la importancia que se recalca en el discurso del programa, de la universidad, de sus familias y sus pares, al rendimiento académico y la responsabilidad que recae sobre ellos, por lo cual se presenta la sobrevaloración del componente académico y la subestimación de las relaciones interpersonales. Con la importancia que adquiere el valor de la responsabilidad, en el seguimiento de instrucciones y el mantenimiento en el programa, se refuerza la importancia asignada a los mensajes provenientes de Ser Pilo Paga, alimentándose el rol pasivo de los estudiantes en torno a la comunicación y el escaso interés en establecer relaciones ascendentes y horizontales que se desliguen del componente académico, pues su prioridad recae en la permanencia (para otros llamada «supervivencia») en el programa para culminar sus estudios sin que el crédito condonable se convierta en una deuda para los estudiantes y sus familias, ya que la pérdida de la calidad de estudiante implica para los Pilos la devolución del dinero que han recibido por parte del Estado.

Considerando que parte de la cultura organizacional se manifiesta en las relaciones, comportamientos e interacciones observables, como un código que orienta las prácticas sociales que unen a las organizaciones, desde el cual se identifican asuntos más profundos como las creencias y valores que las sostienen (Rodríguez, 2009; Toca \& Carrillo, 2009), las características comunicacionales se integran con la construcción de la cultura organizacional de Ser Pilo Paga, al entenderla como un sistema simbólico colectivo que orientan los actos y la configuración de significados por parte de sus miembros, reconociendo los sistemas que la atraviesan y la construcción dinámica propia de las interacciones que se dan en su interior (Allaire \& Firsirotu, 1992; Gonnet, 2012).

De este modo, para demarcar la cultura organizacional relucen, como eje central, las interpretaciones que construyen y los significados que atribuyen los miembros de la organización a las interacciones y experiencias asociadas con la actividad interna y circundante, evidenciándose el valor de la cultura de la organización como construcción subjetiva y dinámica (García, 2006; Smircich, 1983; Leyva, 2007; Gonnet, 2012; Tomàs \& Rodríguez, 2009). Es así como la comunicación se entrelaza con la configuración y fortalecimiento de la cultura organizacional, pues se ven mediadas entre sí en un proceso en que son interdependientes (Scheinsohn, 1993), donde en el programa Ser Pilo Paga se priorizan los comportamientos comunicacionales de los estudiantes como sujetos receptivos y no como agentes activos y generadores de interacciones para fomentar la construcción conjunta de significados (Ordeix, 2009), con lo que se establecen igualmente las características de su cultura, pues desde «la propia cotidianidad va formando la cultura a través de los integrantes de las organizaciones» (Pérez, 2009, p. 188).

La cultura organizacional configurada por los Pilos se ha basado en interacciones escasas, valorando el rendimiento académico que depende poco del relacionamiento entre los estudiantes Pilos con las dependencias de la universidad o con los demás integrantes del programa; además, las normativas en las que se basaron resaltaron sus deberes con el programa respecto a su desempeño académico, convirtiéndose en motivo para atender en Ser Pilo Paga al igual que las responsabilidades establecidas en el ámbito personal y familiar.

La comunicación no es considerada por los miembros del programa como un elemento de gran importancia para el logro de sus objetivos, ya que el rendimiento académico resulta la prioridad que se resuelve 
principalmente a partir del compromiso individual; la interacción con los demás, tanto con pares como con superiores, se llega a establecer en los casos que es necesario para que estos intereses académicos se logren.

Si bien los Pilos han considerado el hecho de pertenecer al programa, ha sido forma semejante a lo que creían que era ser un estudiante universitario regular, con la única distinción del beneficio económico otorgado por el programa, reconocieron que el estudiante Pilo ha asociado directamente las responsabilidades, motivaciones y presiones que pudieron surgir en lo académico con las consecuencias económicas que podrían afectar su futuro y el de su familia. Este asunto ha incidido en la forma de ubicar su rol en la universidad y en el programa, visualizarse a sí mismos, y representar las prioridades, los intereses, los imaginarios y las relaciones que ocurrieron durante su experiencia como Pilos. En vista de ello, tanto estudiantes como trabajadores reconocieron la carencia de interacciones entre los pares, miembros del programa, lo que dificultó la cohesión y la posibilidad de posicionarse como una sociedad particular dentro de la comunidad universitaria. Los intereses de los Pilos se han limitado a enfocarse en la responsabilidad académica y sus efectos económicos, sin llegar a considerar la comunicación ni las interacciones con el programa, ni sus otros miembros como un factor de importancia que los identifique y diferencie de los estudiantes no adscritos.

\section{DISCUSIÓN}

La comunicación interna en la sede Medellín de la Universidad Nacional de Colombia tuvo mayor presencia a partir de los medios digitales, la cual se enfocó en apoyar los ejes misionales de la universidad desde la Unidad de Medios de Comunicación (Unimedios), que se ha encargado del manejo de la imagen institucional y establecer los lineamientos comunicacionales para los contenidos producidos desde cada dependencia, sin que ello significara un control o centralización de la comunicación, ya que su rol de acción se asemejó a la consultoría hecha a partir requerimientos particulares.

En el sistema de Bienestar Universitario de la sede universitaria objeto de investigación, la comunicación se ha determinado a partir de los acuerdos establecidos por la profesional en comunicaciones con las áreas que conforman la dependencia, acogiéndose no sólo a las necesidades de relacionarse con sus públicos de interés, sino también a las características de los programas, las posturas de los jefes de cada área y del jefe del sistema de Bienestar Universitario; todo ello, considerando los lineamientos establecidos por Unimedios en el manejo de la imagen institucional y uso de los medios. Sin embargo, debido a la cantidad de programas ejecutados en cada una de las áreas de esta dependencia, el quehacer del comunicador no logró abarcar cada programa en detalle, significando, para Ser Pilo Paga, su funcionamiento en la sede sin la presencia de un profesional que planificara la comunicación interna con sus estudiantes para la comprensión organizacional del programa y actuar en su desarrollo.

Debido a la complejidad de las universidades, el estudio de su comunicación debe tener en cuenta que su modelo organizacional no se asemeja a otro tipo de agrupación humana (Abadía \& Vaca, 2013), para lo cual sus objetivos y la heterogeneidad de sus públicos amplían el espectro de análisis sobre el lugar de la comunicación en las universidades y su responsabilidad a la hora de alcanzar un consenso en torno a la identidad y la cultura organizacional, enfrentándose a un contexto altamente variable y complejo que ya no se circunscribe a cambios locales o nacionales, sino globales (Tomàs \& Rodríguez, 2009; Tomàs, Armengol, Borrell, Castro, Esteve, Feixas, Gairín, \& Marquès, 2001).

Las interacciones realizadas con el sistema de Bienestar Universitario hacia los estudiantes Pilos buscaban orientar su servicio hacia la 
configuración de relaciones cercanas, privilegiando la comunicación cara a cara, para llevar a una mayor retroalimentación desde una postura informativa, aclaratoria y educativa, lo cual se ha realizado solo con los estudiantes que han presentado interés y disponibilidad para participar de las actividades a las que son invitados, por lo que su disposición fue un factor fundamental para establecer este contacto.

La comunicación cara a cara, a diferencia del correo electrónico, ha contado con mayor riqueza en la información transmitida debido a su expresividad y espontaneidad, potenciando una mayor interacción y alta calidad en la retroalimentación, así como el desarrollo de mayor confianza entre los agentes del proceso (Šárka, 2014; PreciadoHoyos \& Etayo-Pérez, 2014). Sin embargo, este tipo de comunicación no siempre ha sido posible, lo cual invita a pensar en el establecimiento de otros mecanismos que permitan la interacción activa entre las partes, la construcción de confianza y la espontaneidad del flujo, que fomenten por el establecimiento de diálogos y no de la expresión de monólogos, así como un mayor impacto a partir de las emociones y el involucramiento de más sentidos en el proceso comunicativo.

Con ausencia de un plan de comunicaciones para Ser Pilo Paga, si bien se concibió la comunicación como un elemento vital para el funcionamiento del programa, no se visualizó una planeación que estructurara los objetivos y articulara sus actividades con los públicos de interés, llevando a considerar, por parte de algunos empleados, el rol de la comunicación como un ejercicio de apoyo frente al diseño de piezas gráficas para enviar a los públicos y no como un factor a la hora de tomar decisiones o planificar acciones. Es así como la falta de seguimiento, control y evaluación de la comunicación interna en los programas que operan en la universidad, afectan en el posicionamiento y legitimación de la comunicación como elemento vital para el eje estratégico de toda organización, así como vector fundamental de competitividad en el área en que se desempeña (Abadía \& Vaca, 2013; Álvarez-Nobell \& Lesta, 2011).

Asimismo, es un reto para la comunicación organizacional, principalmente en la integración de los medios digitales en sus formas de relacionamiento con los públicos de interés, lograr su compromiso con los objetivos y misión de la organización, propendiendo por el involucramiento de sus miembros (Moreno, Molleda, Athaydes, Suárez, Herrera, \& Álvarez, 2017).

Así, la comunicación en la universidad puede ubicarse como un tema de suma importancia para su componente estratégico, pues, al pensarse más allá del uso de medios, se convierte en responsable de la participación e integración de sus miembros (Herranz, Tapia \& Lázaro, 2009), ya que se cuestionan los flujos de la comunicación y la función de la bidireccionalidad, no solo para que los miembros de la comunidad universitaria se conozcan e informen, sino para propender por la toma de decisiones con base en lo que fluye y se recibe por las estancias superiores (Palencia-Lefler, 2008).

De este modo, un flujo comunicacional con prevalencia descendente unidireccional instaura dinámicas de relacionamiento e interacción entre los públicos de la universidad y del programa, delimitando información, posibilitando y restringiendo accesos, y dando mayor relevancia a unos agentes sobre otros en el proceso comunicativo. Así, la cultura organizacional se relaciona de mayor manera con la comunicación desde las dinámicas, comportamientos e interacciones que se privilegian 0 que se limitan en la comunicación que a la hora de ser declarada en los contenidos emitidos de manera oficial. En el caso de la comunicación vertical, con dinámicas excluyentes de interacción entre las partes implicadas, emergen afectos relacionados con la inconformidad, el temor, la desconfianza y la frustración a la hora de pensar la participación para la toma de 
decisiones y la planeación (Cabra, 2008), lo cual invita a cuestionar la congruencia percibida entre lo que se declara respecto a la cultura de la organización y su expresión activa en las formas como la comunicación se presenta.

Si se concibe una relación estrecha y de mutua influencia entre la comunicación y cultura de una organización, los individuos y las relaciones que se generan entre ellos son elementos vitales para la construcción de significados, comportamientos y sentidos, pues las características de la comunicación, sus contenidos, el flujo de información que ocurren a partir de las interacciones en la universidad, las relaciones entre los imaginarios y las acciones grupales, son elementos que integran tanto lo humano como lo administrativo de la organización (Leyva, 2007). De ese modo, la comunicación dentro de la organización se encarga de estructurar y dinamizar su cultura, la cual, a su vez, legitima las características de la comunicación, estableciéndose una relación de interdependencia a la hora de ser pensadas (Scheinsohn, 1993).

En las organizaciones se evidencian relaciones en torno a los lenguajes utilizados, límites, términos de inclusión y de exclusión, relaciones de poder y jerarquía, intimidad, emociones y relaciones, así como normativas e ideologías que permiten integrarse a partir de la comunicación para funcionar como sistema social (Pedraza, Obispo, Vásquez, \& Gómez, 2015). De este modo, la cultura es construida en las organizaciones en torno a las relaciones, experiencias y significados, definidos en torno a sus espacios, agentes implicados y el devenir de su historia, dándose la posibilidad de transformación no solo por los contenidos declarados, sino por medio de las formas como la comunicación se presenta y establece mecanismos de interacción.

\section{CONCLUSIONES}

Las universidades, como organizaciones complejas que se encuentran atravesadas por diversos sistemas y cobijan públicos con intereses y características diversas, requieren para su estudio a profundidad del reconocimiento de cada una de estas agrupaciones e individuos con los cuales interactúa sin limitarse a su relación laboral con la organización. De este modo, ubicar a los estudiantes como público activo que incide en su funcionamiento $y$ en el cumplimiento de los objetivos misionales de la universidad, amplía el panorama investigativo al indagar por su rol dentro de la organización, así como los sentidos y consecuencias que emergen de las relaciones con este público a partir de la comunicación y cultura organizacionales.

Así, la universidad se resignifica de escenario generador de conocimiento y precursor de investigación, a objeto de estudio para pensar la sociedad y las interacciones que tienen lugar en su interior, debido a que desde su abordaje se fomenta a la comprensión de dinámicas de interacción que van más allá del componente académico - laboral y que da lugar a pensar en el estudiante como agente activo del proceso de construcción de significados y elemento fundamental en el proceso comunicacional que establece la universidad con sus públicos de interés para el logro de sus ejes misionales.

Una iniciativa de carácter nacional, como Ser Pilo Paga, invita inevitablemente a ser foco de estudio a partir de las dinámicas relacionales que se generan en los diversos sistemas que atraviesan al país, dejando de ser un programa de créditos condonables para ser pensado como un escenario dinámico, generador de significados, de relaciones, de posibilidades, limitaciones y de sentidos que necesitan ser estudiados para llegar a comprender sus incidencias en las dinámicas académicas y sociales en las que las universidades se encuentran inmersas. 
Con ello, el abordaje investigativo en asuntos concernientes con la comunicación organizacional en este escenario académico permite la reflexión teórica y la determinación de nuevos métodos para adentrarse a comprender aún más sobre los contextos que tienen lugar dentro de las universidades. El programa Ser Pilo Paga, de este modo, opera más allá de una figura administrativa que surge como iniciativa del Gobierno Nacional de Colombia, pues se integra a las dinámicas organizacionales de cada universidad, de su comunicación y su cultura, para introducir nuevas maneras de interacción que merecen ser estudiadas. Esto hace necesario preguntarse para futuros estudios cómo opera el programa en cada universidad, en cómo puede llegar a incidir el componente público o privado de estas instituciones y cómo se transforman los procesos de significación a partir de los cambios que va teniendo un programa como Ser Pilo Paga en las cohortes, modos de funcionamiento y opiniones de la comunidad.

Es así como esta investigación reafirma la complejidad que puede adquirir una organización como la universidad, siendo un escenario propicio para estudios que se enfoquen en la variedad de las dinámicas que se generan en su interior, no solo por los programas como Ser Pilo Paga o los procesos administrativos que la atraviesan, los cuales se ven afectados por el contexto social, económico, político y cultural de cada país, sino desde el reconocimiento de los agentes que hacen parte de las interacciones y cómo ellas inciden en las representaciones de los individuos y la sociedad. Los estudiantes, de este modo, surgen como figuras fundamentales a la hora de indagar por los procesos dentro de las universidades, siendo su voz y sus experiencias la base para trascender los estudios de la comunicación y la cultura organizacional de las relaciones exclusivamente laborales.

\section{AGRADECIMIENTOS}

Esta investigación se realizó en el marco de la Maestría en Comunicaciones de la Universidad de Antioquia, con el apoyo del Fondo de Becas de Maestría de la Universidad de Antioquia. Asimismo, fue vital el apoyo otorgado por la Sección de Acompañamiento Integral y Bienestar Universitario de la Universidad Nacional de Colombia, sede Medellín, también del personal administrativo, trabajadores $y$ estudiantes que aportaron con sus experiencias al desarrollo del proceso investigativo.

\section{REFERENCIAS}

Abadía, H., \& Vaca, A. (2013). La auditoría de comunicación en universidades privadas del eje cafetero. Revista Académica e Institucional Páginas de la UCP, 94, 89-100. Recuperado de https://dialnet.unirioja.es/servlet/artic ulo?codigo $=5402574$

Allaire, Y., \& Firsirotu, M. (1992). Teorías sobre la cultura organizacional. En Abravanel, H., Allaire, Y., Firsirotu, M., Hobbs, B., Poupart, R., \& Simard, J. Cultura Organizacional: aspectos teóricos, prácticos y metodológicos. Bogotá: Legis. Fondo Editorial.

Álvarez-Nobell, A., \& Lesta, L. (2011). Medición de los aportes de la gestión estratégica de comunicación interna a los objetivos de la organización. Palabra Clave, 14(1), 11-30.

Bartels, J., Peters, O., Jong, M. Pruyn, A., \& van der Molen, M. (2010). Horizontal and vertical communication as determinants of profesional and organisational identification. Personnel Review, 39(2), 210-226. doi: $10.1108 / 00483481011017426$ 
Blanco, N., \& Pirela, J. (2016). La complementariedad metodológica. Espacios Públicos, 19(45), 97-111.

Cabra, N. (2008). Las voces de la organización. Signo y Pensamiento, 52, 165-177.

Constantin, E., \& Constantin, C. (2015). Employee voice - Key factor in internal communication. Procedia - Social and Behavioral Sciences, 191, 975-978. doi: 10.1016/jsbpro.2015.04. 319

Fajardo, L. (2009). A propósito de la comunicación verbal. Forma y Función, 22(2), 121-142.

García, C. (2006). Una aproximación al concepto de cultura organizacional. Univ. Psychol, 5(1), 163-174.

García, J., Ruiz, A., \& Ventura, R. (1999). La auditoría de comunicación interna: una aproximación conceptual y metodológica. Revista Latina de Comunicación Social, 2(18), 1-8.

Gonnet, J. (2012). Cultura, organizaciones y antropología. Una revisión crítica. AVÁ Revista de Antropología, 21, 151-170.

Hernández-Sampieri, R., Fernández, C., \& Baptista, M. (2014). Metodología de la investigación, (6a ed.). México D.F.: Mc Graw Hill.

Herranz, J. M., Tapia, A., \& Lázaro, A. V. (2009). La comunicación interna en la universidad. Investigar para conocer a nuestros públicos. Revista Latina de Comunicación Social, 64, 262-274.

Karanges, E., Johnston, K., Beatson, A., \& Lings, I. (2015). The influence of internal communication on employee engagement: A pilot study. Public Relations Review, 41(1), 129-131. doi: 10.1016/j.pubrev.2014.12.003
Leyva, C. (2007). Bases teóricometodológicas para un enfoque de la gestión de la cultura organizacional en las sedes universitarias municipales. ACIMED, 16(2). 1-15.

Ministerio de Educación Nacional. (2015). Reglamento Operativo Ser Pilo Paga 1. Recuperado de http://documents.tips/documents/regl amento-operativo-ser-pilo-paga.html

Moreno, A., Molleda, J. C., Athaydes, A., Suárez, A. M., Herrera, M., \& Álvarez, A. (2017). Latin American Communication Monitor 2016 - 2017. Tendencias en comunicación estratégica: big data, automatización, engagement, influencers, coaching y competencias. Resultados de una encuesta en 17 países. Madrid, España: EUPRERA/DIRCOM.

Ordeix, E. (2009). Los valores de la organización y el público interno. En M.C. Carretón (Ed.), Las relaciones públicas en la gestión de la comunicación interna (pp.55-66). Alicante: AIRP (Asociación de Investigadores en Relaciones Públicas).

Palencia-Lefler, M. (2008). La incomunicación interna en la universidad española. Revista Latina de Comunicación Social. 63, 277-286.

Pedraza, A. L., Obispo, S. K., Vásquez, G. L., \& Gómez, G. L. (2015). Cultura organizacional desde la teoría de Edgar Schein: estudio fenomenológico. Clío América, 9(17), 17-25.

Pérez, A. (2009). Cultura organizacional: algunas reflexiones a la luz de los nuevos retos. Revista Venezolana de Gerencia (RVG). 46, 183-194.

Preciado-Hoyos, A. \& Etayo-Pérez, C. (2014). Influencia del estilo directivo en la comunicación interna de las 
organizaciones. Una aplicación a las agencias de publicidad. Palabra Clave. 17(2), 412-455.

Resolución No. 101 de 2016. Por la cual se define la política de comunicación de la Universidad Nacional de Colombia y se dictan otras disposiciones, Bogotá, Colombia, 10 de febrero de 2016.

Rodríguez, R. (2009). La cultura organizacional. Un potencial activo estratégico desde la perspectiva de la administración. Invenio, 12(22), 67-92.

Ruck, K. \& Welch, M. (2012). Valuing internal communication; management and employee perspectives. Public Relations Review, 38(2), 294-302. doi: 10.1016/j.pubrev.2011.12.016

Saladrigas, H. (2005). Comunicación organizacional: Matrices teóricas y enfoques comunicativos. Revista Latina de Comunicación Social, 60, 1-13. Recuperado de http://www.ull.es/publicaciones/latina/ 200540salabrigas.pdf

Šárka, H. (2014). Tools of Internal Communication from Knowledge Transfer Perspective. Journal of Competitiveness, 6(4), 50-62. doi: 10.7441/joc.2014.04.04

Scheinsohn, D. (1993). Comunicación estratégica: Management $\quad y$ fundamentos de la imagen corporativa. Bogotá: Ediciones Macchi.

Senior Naveda, A., Colina, J., Marín, F., \& Perozo, B. (2012). Visión complementaria entre los métodos cualitativos y cuantitativos en la investigación social. Una aproximación teórica. Multiciencias, 12, 106-114.

Smircich, L. (1983). Conceptos de cultura y análisis organizacional (Versión traducida al español). Administrative Science Quarterly, 28(3), 339-358.

Tapia, A. \& Matellanes, M. (2009). Las herramientas de comunicación interna en la Universidad. En M.C. Carretón (Ed.), Las relaciones públicas en la gestión de la comunicación interna. Alicante: AIRP (Asociación de Investigadores en Relaciones Públicas).

Toca, C. \& Carrillo, J. (2009). Asuntos teóricos y metodológicos de la cultura organizacional. Civilizar, 9(17), 117-136.

Tomàs, M., Armengol, C., Borrell, N., Castro, D., Esteve, J., Feixas, M., Gairín, J., \& Marqués, P. (2001). El cambio de la cultura en las universidades del siglo XXI. Educar, 28, 147-162.

Tomàs, M. \& Rodríguez, D. (2009). Conocer la cultura de la universidad contemporánea: el CICOU. Revista Iberoamericana de Educación, 49, 1-25. 